\title{
Una domanda azzeccata
}

\section{Francesco Avolio}

PUBBLICATO: 30 OCTOBER 2020

\section{Quesito:}

Un lettore di Napoli chiede se l'uso del verbo azzeccare nel significato di 'aderire', 'attaccare' sia da ritenersi dialettale; una lettrice dalla provincia di Firenze domanda chiarimenti sull'uso dello stesso verbo, da lei conosciuto come equivalente di indovinare, in espressioni come ma che ci azzecca?, non ci azzecca niente; infine un lettore dalla Svizzera domanda se vi sia differenza tra azzeccare e indovinare.

\section{Una domanda azzeccata}

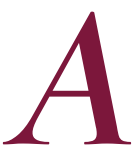
$z z e c c a ̀(r e)$ in molti dialetti centro-meridionali significa 'colpire (nel segno)'. Ad esempio per l'Abruzzo (Lanciano, Chieti) Gennaro Finamore riporta, nel I893, il seguente esempio: J'á 'zzeccate ' $m$ bètte 'l'ha colpito nel petto'. Da qui al senso di 'fare centro', e quindi 'indovinare' il passaggio è breve. Impossibile, a questo riguardo, non ricordare il famoso personaggio manzoniano dell'avvocato Azzeccagarbugli, in realtà un leguleio il cui soprannome sarebbe dovuto all'unione tra azzeccare 'indovinare' e garbugli 'imbrogli, cose non giuste', quindi 'indovinare cose non giuste'. Tuttavia, il (sopran)nome (che ha un precedente di rilievo in Machiavelli, la cui opera era ben nota al Manzoni) sarebbe invece un'italianizzazione del termine dialettale milanese zaccagarbij, che Francesco Cherubini (Cherubini I839-56, IV, s. v.) traduce 'scioglitore di nodi'. Lorigine del verbo viene comunque rintracciata da alcuni (Devoto i968; DELI, s. v. azzeccàre) nel medio alto tedesco zecken 'colpire, menare un colpo', mentre per altri (DEDI, s. v. azzëccùso) l'etimologia è tuttora discussa.

Dal senso di 'colpire' si può arrivare senza troppa difficoltà anche a quello di 'attaccare', verbo che nella stessa lingua italiana ha varie accezioni, da 'aggredire' a 'congiungere' e 'incollare': si attacca qualcosa dando spesso dei piccoli colpi (ad esempio col martello sul chiodo). Da qui il napoletano e meridionale azzeccare, sia nel significato di 'congiungere una cosa con l'altra', 'attaccare', 'appiccicare', sia in quello di 'avvicinarsi', quasi attaccandosi a qualcun altro (D’Ambra I873, s. v. Azzeccare; e infatti azzëcúsë si dice spesso di una persona che non si leva mai di torno). A questo significato potrebbe ricollegarsi il nome della zecca (acaro che, com'è noto, si appiccica alla pelle dei cani, di altri animali e perfino dell'uomo, succhiandone il sangue), anch'esso del resto derivante, secondo Devoto, dal germanico, e in particolare dal longobardo zëkka (cfr. anche DELI, V, s. v. zécca ${ }^{\mathrm{I}}$ ). Azzeccare può poi assumere - ad esempio in alcune parlate dell'Italia mediana - il senso di 'salire', con uno sviluppo semantico analogo a quello dell'italiano giungere (passato da 'congiungere' ad 'arrivare', cioè 'unirsi ad un luogo').

Da 'congiungere qualcosa a qualcos'altro', vale a dire 'entrare in relazione', si passa infine ad azzeccare nel senso di 'entrarci': che c'azzécca? - espressione che negli anni Novanta divenne celebre grazie ad Antonio Di Pietro (molisano di Montenero di Bisaccia, $\mathrm{Cb}$ ) - vuol dire quindi 'che c'entra?', ossia 'che rapporto c'è con quello che stai dicendo?'.

Il ventaglio di significati di questo verbo oggi assai comune mostra una volta di più la rilevanza del ruolo svolto, ormai da molto tempo, dai nostri dialetti (in questo caso soprattutto centromeridionali) nella continua opera di arricchimento e rinnovamento semantico e lessicale della lingua italiana. 
Nota bibliografica:

- Cherubini 1839-56 = Francesco Cherubini, Vocabolario milanese-italiano, Milano, Imperial Regia Stamperia, poi Società Tipografica dei Classici Italiani, I839-I856 (5 voll.).

- DEDI = Manlio Cortelazzo, Carla Marcato, Dizionario etimologico dei dialetti italiani (DEDI), Torino, Utet Libreria, 2005.

- D'Ambra 1873 = Raffaele D'Ambra, Vocabolario napolitano-toscano domestico di arti e mestieri, Napoli, presso l'autore, 1873 .

- Devoto 1968 = Giacomo Devoto, Avviamento alla etimologia italiana, Firenze, Le Monnier, I968.

- Finamore 1893 = Gennaro Finamore, Vocabolario dell'uso abruzzese, Città di Castello, Tipografia Lapi, I893.

\section{Cita come:}

Francesco Avolio, Una domanda azzeccata, "Italiano digitale", 2020, XV, 2020/4 (ottobre-dicembre) DOI: $10.35948 / 2532-9006 / 2020.4416$

\section{Copyright 2020 Accademia della Crusca}

Pubblicato con licenza creative commons CC BY-NC-ND 